\title{
A New PET Scanner with Semiconductor Detectors Enables Better Identification of Intratumoral Inhomogeneity
}

Tohru Shiga ${ }^{1}$, Yuichi Morimoto ${ }^{2}$, Naoki Kubo ${ }^{3}$, Norio Katoh ${ }^{4}$, Chietsugu Katoh ${ }^{3}$, Wataru Takeuchi ${ }^{2}$, Reiko Usui ${ }^{1}$, Kenji Hirata ${ }^{1}$, Shinichi Kojima ${ }^{2}$, Kikuo Umegaki ${ }^{2}$, Hiroki Shirato ${ }^{4}$, and Nagara Tamaki ${ }^{1}$

${ }^{1}$ Department of Nuclear Medicine, School of Medicine, Hokkaido University, Sapporo, Hokkaido, Japan; ${ }^{2}$ Hitachi Ltd. Central Research Laboratory, Hitachi, Ibaraki, Japan; ${ }^{3}$ Faculty of Health Science, Hokkaido University, Sapporo, Hokkaido, Japan; and ${ }^{4}$ Department of Radiology, School of Medicine, Hokkaido University, Sapporo, Hokkaido, Japan

An autoradiography method revealed intratumoral inhomogeneity in various solid tumors. It is becoming increasingly important to estimate intratumoral inhomogeneity. However, with low spatial resolution and high scatter noise, it is difficult to detect intratumoral inhomogeneity in clinical settings. We developed a new PET system with CdTe semiconductor detectors to provide images with high spatial resolution and low scatter noise. Both phantom images and patients' images were analyzed to evaluate intratumoral inhomogeneity. Methods: This study was performed with a cold spot phantom that had 6-mm-diameter cold sphenoid defects, a dual-cylinder phantom with an adjusted concentration of 1:2, and an " $H$ "-shaped hot phantom. These were surrounded with water. Phantom images and ${ }^{18} \mathrm{~F}-\mathrm{FDG}$ PET images of patients with nasopharyngeal cancer were compared with conventional bismuth germanate PET images. Profile curves for the phantoms were measured as peak-to-valley ratios to define contrast. Intratumoral inhomogeneity and tumor edge sharpness were evaluated on the images of the patients. Results: The contrast obtained with the semiconductor PET scanner (1.53) was $28 \%$ higher than that obtained with the conventional scanner (1.20) for the 6-mm-diameter cold sphenoid phantom. The contrast obtained with the semiconductor PET scanner (1.43) was $27 \%$ higher than that obtained with the conventional scanner (1.13) for the dual-cylinder phantom. Similarly, the $2-\mathrm{mm}$ cold region between $1-\mathrm{mm}$ hot rods was identified only by the new PET scanner and not by the conventional scanner. The new PET scanner identified intratumoral inhomogeneity in more detail than the conventional scanner in 6 of 10 patients. The tumor edge was sharper on the images obtained with the new PET scanner than on those obtained with the conventional scanner. Conclusion: These phantom and clinical studies suggested that this new PET scanner has the potential for better identification of intratumoral inhomogeneity, probably because of its high spatial resolution and low scatter noise.

Received Jun. 2, 2008; revision accepted Sep. 8, 2008.

For correspondence or reprints contact: Tohru Shiga, Department of Nuclear Medicine, School of Medicine, Hokkaido University, Kita-15, Nishi-6, Sapporo, Hokkaido, Japan 060.

E-mail: tshiga@med.hokudai.ac.jp

COPYRIGHT @ 2009 by the Society of Nuclear Medicine, Inc.
Key Words: oncology; PET; semiconductor; intratumoral heterogeneity

J Nucl Med 2009; 50:148-155

DOI: 10.2967/jnumed.108.054833

$\mathbf{P}_{\mathrm{H}}$ ET with ${ }^{18} \mathrm{~F}-\mathrm{FDG}$ has been widely used in oncology studies. A high-resolution PET camera permits precise evaluation of tumor localization and treatment effects. Recently, CdTe semiconductors were used for the direct conversion of $\gamma$-rays without scintillator material $(1,2)$. High energy resolution and flexibility in both the sizing and the fine arrangement of detectors are expected to improve image quality. These characteristics of semiconductor detectors may also lead to improved PET images because the high energy resolution offers a reduction in scatter noise, like that seen with $\gamma$-camera and SPECT applications $(1,3)$.

A depth-of-interaction (DOI) detection system has already been used in some PET scanners, particularly in PET scanners dedicated to use for the human brain. This is because DOI information is very useful for reducing the parallax errors at the periphery of the field of view (FOV) $(4,5)$. With both semiconductor detectors and a DOI system, high-quality PET images with low scatter noise and high spatial resolution are expected.

Intensity-modulated radiation therapy is an advanced mode of highly precise radiotherapy that uses computercontrolled $\mathrm{x}$-ray accelerators to deliver precise radiation doses to a malignant tumor or specific areas within a tumor; intensity-modulated radiation therapy has become widely used in clinical settings (6-8). We reported that image fusion between ${ }^{18} \mathrm{~F}-\mathrm{FDG}$ PET and MRI/CT was useful for gross tumor volume and clinical target volume determinations in conformal radiation therapy (11), and several other studies revealed the clinical impact of ${ }^{18} \mathrm{~F}-\mathrm{FDG} \mathrm{PET} / \mathrm{CT}$ fusion on radiation planning $(8-10)$. In radiation therapy, it is becoming increasingly important to estimate intratumoral inhomogeneity for more accurate radiation planning. 
We developed a new PET scanner with CdTe semiconductor detectors and a DOI system. Both phantom images and patients' images obtained with this new PET scanner were compared with conventional bismuth germanate PET images to evaluate intratumoral inhomogeneity.

\section{MATERIALS AND METHODS}

\section{Outline of Our New PET Scanner}

Figure 1 shows our prototype 3-dimensional (3D) PET scanner dedicated to human brain imaging. The diameter of the patient port is $350 \mathrm{~mm}$, the transaxial FOV is $310 \mathrm{~mm}$, and the axial FOV is $250 \mathrm{~mm}$. There are 18 detector units radially arranged around the patient port.

Figure 2 shows the structure of the detector unit. The new detector size is $2 \times 4 \times 7.5 \mathrm{~mm}$. The dimensions of the detector unit are $100 \times 400 \times 350 \mathrm{~mm}$. In the unit, the detector boards are arranged in parallel, and the detectors are mounted on both sides of each board. A detector board has 96 detectors on each side (192 detectors in total) and signal processors. These processors include applicationspecific integrated circuits mounted along the incident direction of $\gamma$-rays. Signals are read by a 3-layer DOI system. Each unit has 22 boards and about 4,000 detectors. The entire system is cooled by forced air.

CdTe detectors have good energy resolution characteristics for use at room temperature. However, it is well known that the polarization effect deteriorates the energy resolution in several tens of minutes and then shifts the photopeaks. For stabilization of detector performance, an automatic periodic bias reset technique was developed for the scanner (12). The negligible reset duration $(0.5 \mathrm{~s})$ with a 5 -min reset interval is sufficient to stabilize detector performance under normal operating conditions at room temperature.

\section{Physical Performance of New PET Scanner}

The energy resolution of the new scanner is $4.1 \%$ (full width at half maximum [FWHM]), which is superior to the energy resolution obtained with available scintillation detectors (e.g., 10\%-20\%). The timing resolution was evaluated with a ${ }^{68} \mathrm{Ge}-\mathrm{Ga}$ point radiation source with an activity of $3.7 \mathrm{MBq}$ placed at the center of the FOV. Measurements were taken for $5 \mathrm{~min}$. The timing resolution was found to be $6.8 \mathrm{~ns}$ (FWHM). This value was almost equivalent to that of conventional PET scanners; therefore, we set the time window at $14 \mathrm{~ns}$, a value that is almost the same as that of the ECAT EXACT HR+ scanner (Asahi-Siemens) $(13,14)$.

Physical performance characteristics, such as spatial resolution, sensitivity, and noise equivalent count rates, were measured with

A
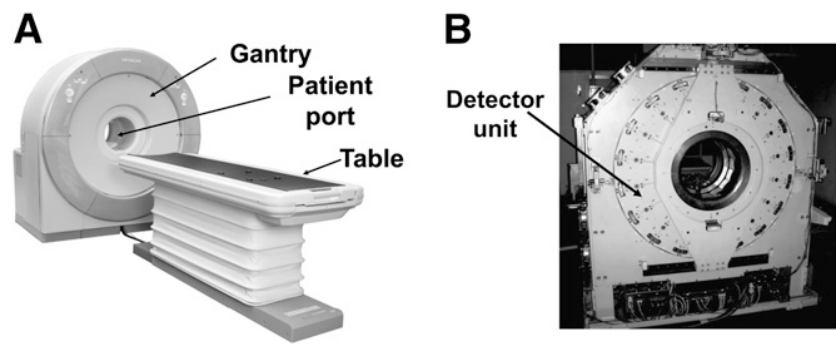

FIGURE 1. (A) Prototype 3D PET scanner dedicated to human brain imaging. Diameter of patient port is $350 \mathrm{~mm}$, transaxial FOV is $310 \mathrm{~mm}$, and axial FOV is $250 \mathrm{~mm}$. (B) Eighteen detector units are radially arranged around patient port.

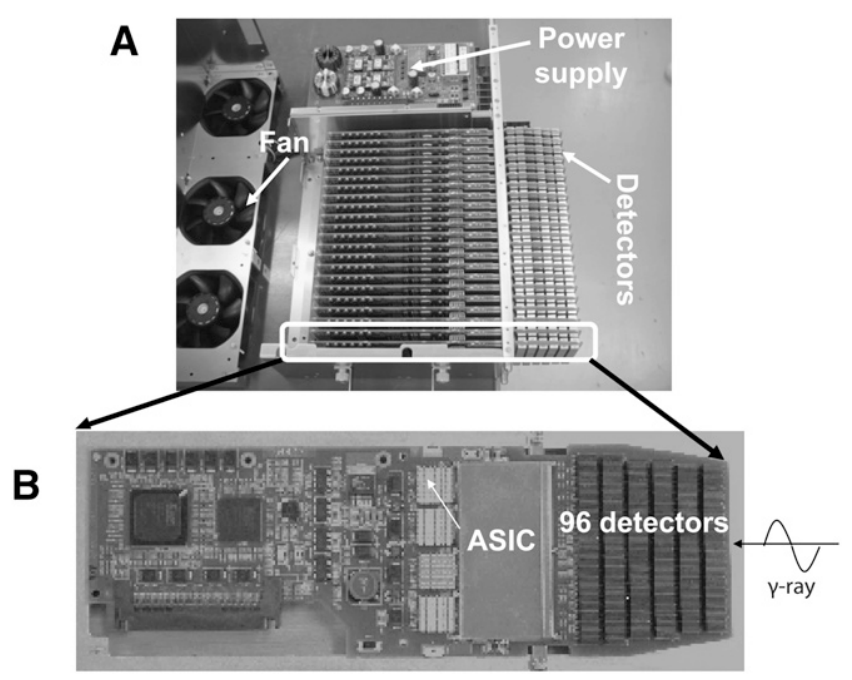

FIGURE 2. (A) Structure of detector unit. New detector size is $2 \times 4 \times 7.5 \mathrm{~mm}$. Dimensions of detector unit are $100 \times 400$ $\times 350 \mathrm{~mm}$. Detector boards are arranged in parallel, and detectors are mounted on both sides of each board. (B) Detector board has 96 detectors on each side (192 detectors in total) and signal processors. These processors include application-specific integrated circuits (ASICs) mounted along incident direction of $\gamma$-rays. Signals are read by 3-layer DOI system. Each unit has 22 boards and about 4,000 detectors. Entire system is cooled by forced air.

National Electrical Manufacturers Association (NEMA) standards. Table 1 summarizes the results of the evaluations. The transverse and axial resolutions near the center are 2.3 and $5.1 \mathrm{~mm}$, respectively. The absolute sensitivity and the scatter fraction of the scanner evaluated with the NEMA NU 2-1994 phantom are $17.6 \mathrm{kcps} / \mathrm{kBq} /$ $\mathrm{mL}$ and $23 \%$, respectively. These estimates are for a lower-energy threshold of $450 \mathrm{keV}$. The NEC2R value is $41 \mathrm{kcps}$ at $3.7 \mathrm{kBq} / \mathrm{mL}$ (15).

\section{Study of Phantoms}

Three different phantom studies were performed.

\section{TABLE 1. Performance of New PET Scanner}

\begin{tabular}{clc}
$\begin{array}{c}\text { Performance index } \\
\text { Spatial resolution } \\
\text { (transaxial) }\end{array}$ & \multicolumn{1}{c}{ Result } & \multicolumn{1}{c}{ Standard } \\
$1 \mathrm{~cm}$ & $2.3 \mathrm{~mm}$ & NEMA NU 2-2001 \\
$10 \mathrm{~cm}$ & $4.8 \mathrm{~mm}$ & NEMA NU 2-2001 \\
$\begin{array}{c}\text { Spatial resolution } \\
\text { (axial) }\end{array}$ & & \\
$1 \mathrm{~cm}$ & $5.1 \mathrm{~mm}$ & NEMA NU 2-2001 \\
$10 \mathrm{~cm}$ & $5.9 \mathrm{~mm}$ & NEMA NU 2-2001 \\
$\begin{array}{c}\text { Sensitivity (true) at } \\
450-530 \mathrm{keV}\end{array}$ & $17.6 \mathrm{kcps} / \mathrm{kBq} / \mathrm{mL}$ & NEMA NU 2-1994 \\
$\begin{array}{c}\text { Scatter fraction (3D) } \\
\text { at } 450-530 \mathrm{keV}\end{array}$ & $23 \%$ & NEMA NU 2-1994 \\
NEC-2R & & \\
(at $450-530 \mathrm{keV})$ & & \\
$3.7 \mathrm{kBq} / \mathrm{mL}$ & $30 \mathrm{kcps}$ & NEMA NU 2-1994 \\
$7.9 \mathrm{kBq} / \mathrm{mL}$ & $41 \mathrm{kcps}$ & NEMA NU 2-1994 \\
Energy resolution & $4.1 \%$ & \\
at $511 \mathrm{keV}$ & & \\
\hline
\end{tabular}


To estimate PET image uniformity, a pool phantom filled with ${ }^{18} \mathrm{~F}$ at $74 \mathrm{kBq} / \mathrm{mL}$ was used. The acquisition time was set at $10 \mathrm{~min}$ for both scanners.

The second phantom, an elliptic phantom measuring $136 \times 188$ $\mathrm{mm}$, consisted of 4 small compartments containing water. The first compartment was a uniform cylinder in the middle, the second compartment had a cylinder with five 6-mm-diameter cold spots, the third compartment had a cylinder with a 25-mm-diameter cold region, and the fourth compartment had a cylinder filled with half the radioactivity of the remaining compartments (Fig. 3A). All of the small compartments were filled with ${ }^{18} \mathrm{~F}$ at $63 \mathrm{kBq} / \mathrm{mL}$. This tumor phantom was used to evaluate image contrast and inhomogeneity.

The third phantom was designed as a tumor phantom containing small hot spots. This "H"-shaped hot phantom was made of 1-mmdiameter capillary tubes filled with ${ }^{18} \mathrm{~F}$ at $198 \mathrm{MBq} / \mathrm{mL}$. The $\mathrm{H}$ shape was $5 \mathrm{~mm}$ high and $4 \mathrm{~mm}$ wide. This phantom was surrounded with water (Fig. 3B).

Images of these phantoms were acquired with our semiconductor PET scanner and with a conventional high-resolution bismuth germanate PET scanner (EXACT HR+; Asahi-Siemens). Profile curves for the phantoms were determined from images obtained with both scanners for quantitative assessment of the cold-spot and hot-spot lesions by calculating the contrast between peak and valley activities. The energy windows of the semiconductor PET and HR+ PET scanners were 490-530 and 350-650 keV, respectively.

\section{Study of Patients}

We studied 10 patients with nasopharyngeal cancer before treatment or suspected of having a local recurrence. The characteristics of the patients are shown in Table 2. The patients gave written informed consent according to the Helsinki II Declaration, and this study was approved by the Ethics Committee of the Hokkaido University School of Medicine.

Before the PET study, all patients fasted for at least $6 \mathrm{~h}$. Serum glucose levels were checked in all of the patients before the administration of ${ }^{18} \mathrm{~F}$-FDG. The dose of ${ }^{18} \mathrm{~F}$-FDG for each patient was $370 \mathrm{MBq}$. Images of the patients were acquired with the semiconductor PET and HR + PET scanners. Two time courses were used in this study. In one time course, the protocol for image acquisition was as follows: HR + PET in whole-body mode, HR+ PET in brain mode, and semiconductor PET. In the other time course, the protocol for image acquisition was as follows: semiconductor PET, HR + PET in brain mode, and HR + PET in wholebody mode (Fig. 4). The time course protocols were randomly selected.

Tumor edge sharpness was evaluated with profile curves.

Two experienced nuclear physicians visually interpreted the images. In addition, intratumoral inhomogeneity was scored from 0 to 4 , as expected from MRI or CT findings, as follows: 0 , no uptake; 1 , most homogeneous; 2 , intermediate; and 3, most inhomogeneous. First, 2 nuclear physicians independently scored the images to determine interobserver variability. Then, these 2 physicians discussed their interpretations to determine a final score.

In addition, an MRI scan was obtained with a 1.5-T scanner (Magnetome Vision or Magnetome Symphony; Asahi-Siemens). Transaxial T1-weighted, T2-weighted, gadolinium-enhanced T1weighted, and fluid-attenuated inversion recovery images were acquired. All images were acquired with a 5-mm slice thickness and no slice gap. Coronal and sagittal images were also acquired in some cases. The findings from ${ }^{18}$ F-FDG PET and MRI were compared.

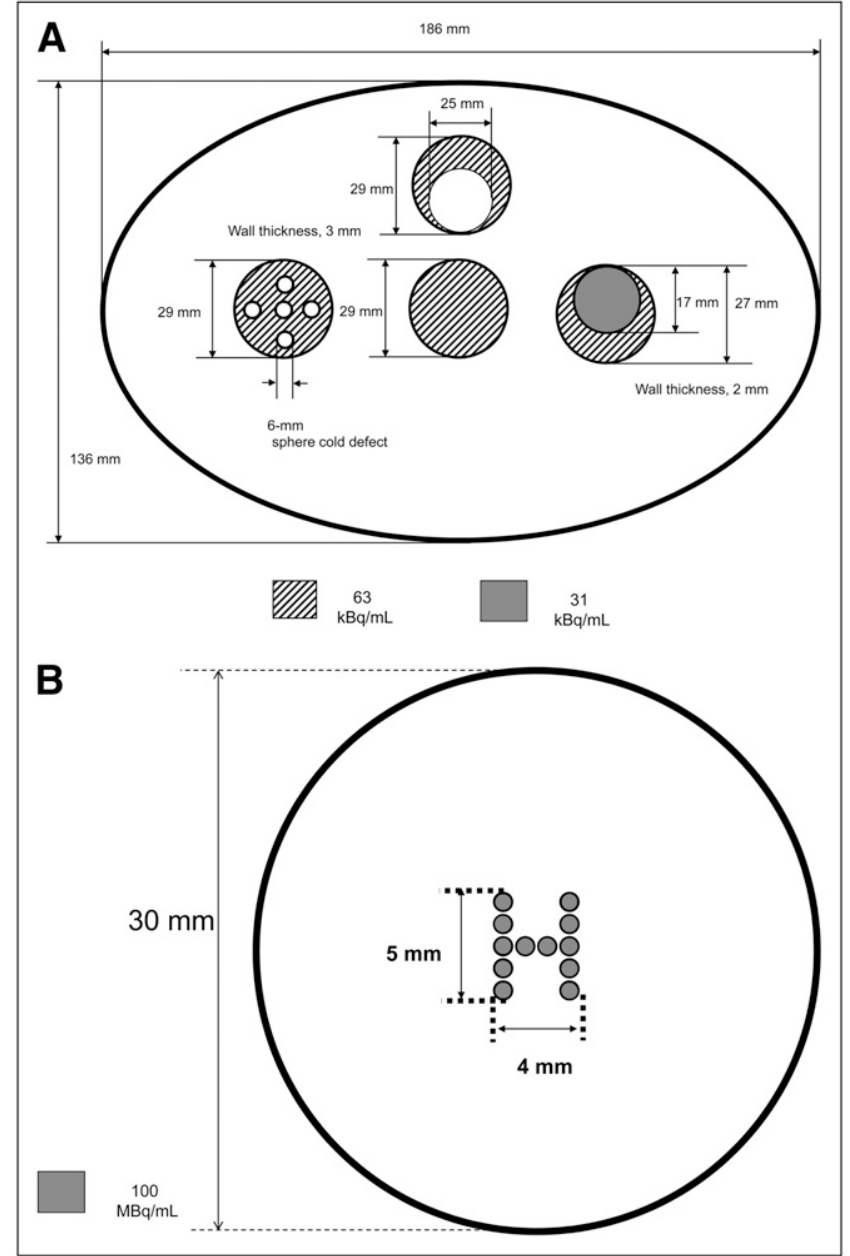

FIGURE 3. Scheme of second elliptic phantom $(136 \times 188$ $\mathrm{mm}$ ) consisting of 4 small compartments $(A)$ and $\mathrm{H}$-shaped hot phantom (B). First compartment had uniform cylinder in middle, second compartment had cylinder with five 6-mm-diameter cold spots, third compartment had cylinder with 25-mm-diameter cold region, and fourth compartment had cylinder filled with half the radioactivity in remaining compartments.

\section{HR + PET in Whole-Body Mode}

Whole-body static emission scanning was performed $60 \mathrm{~min}$ after ${ }^{18} \mathrm{~F}-\mathrm{FDG}$ administration in the 3D acquisition mode for $3 \mathrm{~min}$ per bed position. The energy window was $350-650 \mathrm{keV}$. Thereafter, transmission scanning with externally rotating ${ }^{68} \mathrm{Ge}$ rod sources was performed for attenuation correction. The acquired 3D sinograms were converted into 2-dimensional (2D) sinograms with the Fourier rebinning algorithm, and attenuation-corrected data were reconstructed iteratively by use of an ordered-subset expectation maximization algorithm with 32 subsets and 1 iteration.

\section{$\mathrm{HR}+\mathrm{PET}$ in Brain Mode}

Images were acquired with a 3-min transmission scan and a 10-min emission scan. Ten-minute regional static scanning with the nasopharynx centered was performed with the HR+ PET scanner in the $3 \mathrm{D}$ acquisition mode, and images were reconstructed with the brain mode of the manufacturer's software. The energy window was $350-650 \mathrm{keV}$. The acquired 3D sinograms were converted into 2D 
TABLE 2. Characteristics of Patients

\begin{tabular}{|c|c|c|c|c|c|c|}
\hline Patient & Age (y) & Sex & Pathology & $\begin{array}{c}\text { TNM } \\
\text { classification }\end{array}$ & Comments & $\begin{array}{l}\text { Findings from } \\
\text { primary lesion }\end{array}$ \\
\hline 1 & 76 & $M$ & SCC & T2bN2M0 & Before treatment & $\begin{array}{l}\text { Left nasopharyngeal uptake } \\
\text { extending to left fossa of } \\
\text { Rosenmüller }\end{array}$ \\
\hline 2 & 30 & M & $\begin{array}{l}\text { Undifferentiated } \\
\text { cancer }\end{array}$ & T3N2M0 & Before treatment & $\begin{array}{l}\text { Bilateral nasopharyngeal-oropharyngeal } \\
\text { uptake }\end{array}$ \\
\hline 3 & 67 & $\mathrm{~F}$ & $\begin{array}{l}\text { Undifferentiated } \\
\text { cancer }\end{array}$ & T3N2M0 & Before treatment & $\begin{array}{l}\text { Strong uptake in retronasopharyngeal } \\
\text { space extending to left fossa of } \\
\text { Rosenmüller }\end{array}$ \\
\hline 4 & 61 & M & SCC & T3N1M0 & Before treatment & $\begin{array}{l}\text { Strong uptake in retronasopharyngeal } \\
\text { space extending to right fossa of } \\
\text { Rosenmüller }\end{array}$ \\
\hline 5 & 35 & $\mathrm{~F}$ & SCC & T4N1M0 & Before treatment & $\begin{array}{l}\text { Strong uptake in right nasopharyngeal } \\
\text { space extending to right cavernous } \\
\text { sinus }\end{array}$ \\
\hline 6 & 61 & M & SCC & T1N1M0 & Before treatment & $\begin{array}{l}\text { Uptake along retronasopharyngeal wall } \\
\text { and right fossa of Rosenmüller }\end{array}$ \\
\hline 7 & 53 & M & $\begin{array}{l}\text { Undifferentiated } \\
\text { cancer }\end{array}$ & T1N0M0 & Before treatment & $\begin{array}{l}\text { Uptake along retronasopharyngeal wall } \\
\text { and right fossa of Rosenmüller }\end{array}$ \\
\hline 8 & 71 & $\mathrm{~F}$ & $\begin{array}{l}\text { Poorly differentiated } \\
\text { SCC }\end{array}$ & T4NxM0 & Before treatment & $\begin{array}{l}\text { Strong uptake in right nasopharyngeal } \\
\text { space extending to right cavernous } \\
\text { sinus }\end{array}$ \\
\hline 9 & 44 & M & $\begin{array}{l}\text { Undifferentiated } \\
\text { cancer }\end{array}$ & T4N2M0 & $\begin{array}{l}\text { Local recurrence in } \\
\text { cavernous } \\
\text { sinus suggested } \\
\text { by MRI after } \\
\text { chemoradiation } \\
\text { therapy }\end{array}$ & No uptake \\
\hline 10 & 28 & $\mathrm{~F}$ & SCC & T3N2M0 & $\begin{array}{l}\text { Local recurrence in } \\
\text { clivus suggested } \\
\text { by MRI after } \\
\text { chemoradiation } \\
\text { therapy }\end{array}$ & No uptake \\
\hline
\end{tabular}

sinograms with the Fourier rebinning algorithm. The images were reconstructed by direct-inversion Fourier transformation. The reconstruction filter was a Hanning filter with 4-mm FHWM. The reconstruction matrix was $256 \times 256$, and the FOV was $33 \mathrm{~cm}$ in diameter. The FWHM was $6.4 \mathrm{~mm}$ after reconstruction.

\section{Semiconductor PET}

Images were acquired with a 3-min transmission scan and a 12-min emission scan. Twelve-minute emission scanning was performed in the 3D acquisition mode. Because of the high energy resolution, achieving a reduction in scatter noise required the energy window to be set at 490-530 keV (double FWHM of energy resolution). To compensate for the relatively low sensitivity derived from the narrow window setting and the low level of interaction of 511-keV $\gamma$-rays and CdTe materials, we developed a new reconstruction algorithm by application of the median root prior (MRP), known to be useful for edge preservation. In addition, for the recovery of resolution, a measured point spread function was convolved with images and the system matrix in the maximum a posteriori (MAP) iterative process. The effects of reducing noise and recovering resolution can be controlled by choosing the degree of prior contribution. The proposed reconstruction method improved image quality in terms of statistical noise and resolution. With the choice of a suitable degree of prior contribution, this method can improve the quality of images reconstructed from noisy or sparse data (16). The FWHM was about $2.5 \mathrm{~mm}$ at $1 \mathrm{~cm}$ or $2.9 \mathrm{~mm}$ at $10 \mathrm{~cm}$ in a transaxial slice.

\section{Statistical Analysis}

The means and SDs for intratumoral inhomogeneity values were calculated. The Wilcoxon matched-pair signed rank test was used to determine the significance of score differences between the 2 techniques. A $P$ value of less than 0.05 was considered significant.

\section{RESULTS}

\section{Study of Phantoms}

The profile curves showing the uniformity of phantom images obtained with the semiconductor PET and HR + PET scanners are shown in Figure 5A. The uniformity of the reconstructed images was evaluated on the basis of the SD per average value in a cylindric region of interest (ROI). Cylindric ROIs were set at the center of the phantom. The diameter and height of the ROI were 180 and $120 \mathrm{~mm}$, respectively. The uniformity of the images obtained with the $\mathrm{HR}+\mathrm{PET}$ scanner was 0.15 , and that of the images obtained with the semiconductor PET scanner was 0.13. Thus, uni- 


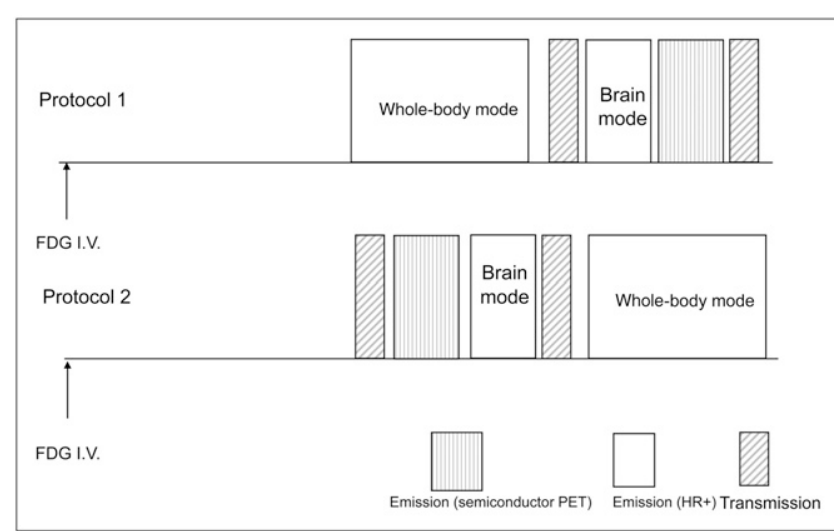

FIGURE 4. Time course protocols. Order in time course protocol 1: HR+ PET in whole-body mode, HR+ PET in brain mode, and semiconductor PET; order in time course protocol 2: semiconductor PET, HR+ PET in brain mode, and HR+ PET in whole-body mode. I.V. = intravenously.

formity was well maintained, and the values were similar for both scanners.

The images of the second phantom showed a $25-\mathrm{mm}$ cold spot clearly for both the semiconductor PET and the HR+ PET scanners. However, the profile curves for the second phantom indicated better contrast for the 6-mm cold sphenoid phantom as well as the dual-cylinder phantom on images obtained with the semiconductor PET scanner. The contrast obtained with the semiconductor PET scanner (1.53) was $28 \%$ higher than that obtained with the HR+ scanner (1.20) for the 6-mm-diameter cold sphenoid phantom. The contrast obtained with the semiconductor PET scanner (1.43) was $27 \%$ higher than that obtained with the $\mathrm{HR}+$ scanner (1.13) for the dual-cylinder phantom (Fig. 5B).

The 1-mm hot rods in the $\mathrm{H}$-shaped hot phantom were detected only by semiconductor PET and not by HR + PET. Similarly, the 2-mm cold region between small hot rods was also identified. The profile curves confirmed the detection of the small hot rods and the cold spot in between because of the increased contrast of the small structures revealed by semiconductor PET (Fig. 5C).

\section{Study of Patients}

For qualitative analysis, semiconductor PET identified intratumoral inhomogeneous glucose metabolism in more detail than HR + PET in 8 of 10 patients (Table 3). In all 6 of these patients, intratumoral inhomogeneity or small structures in the tumor were confirmed by MRI.

The profile curves were sharper for semiconductor PET than for HR + PET in both the brain mode and the wholebody mode for all images from the 8 patients who showed abnormal uptake (Figs. 6 and 7).

Interobserver agreement on the inhomogeneity scores was $100 \%$. The scores for inhomogeneity were higher in the brain mode than in the whole-body mode; however, those for semiconductor PET were significantly higher than those for HR+ PET in the brain mode $(P<0.01)$ (Table 3$)$.

The results for representative patients are shown in Figures 6 and 7. Figure 6 shows the results for a 61-y-old man with nasopharyngeal squamous cell cancer. Semiconductor PET identified intratumoral inhomogeneous glucose metabolism in more detail than HR+ PET. T2-weighted MRI also showed inhomogeneous signal intensity in the primary lesion. The profile curve obtained for the tumor with semiconductor PET was the sharpest among the 3 images shown.

Figure 7 shows the results for a 35 -y-old woman with nasopharyngeal squamous cell cancer. The tumor reached the left cavernous sinus in this patient. Semiconductor PET revealed an area of low uptake in the tumor that corresponded to the internal carotid artery. Gadolinium-enhanced MRI showed that the left internal carotid artery penetrated the tumor. The profile curve obtained for the tumor with semiconductor PET was sharper than those obtained with HR+ PET in the brain mode and the whole-body mode.

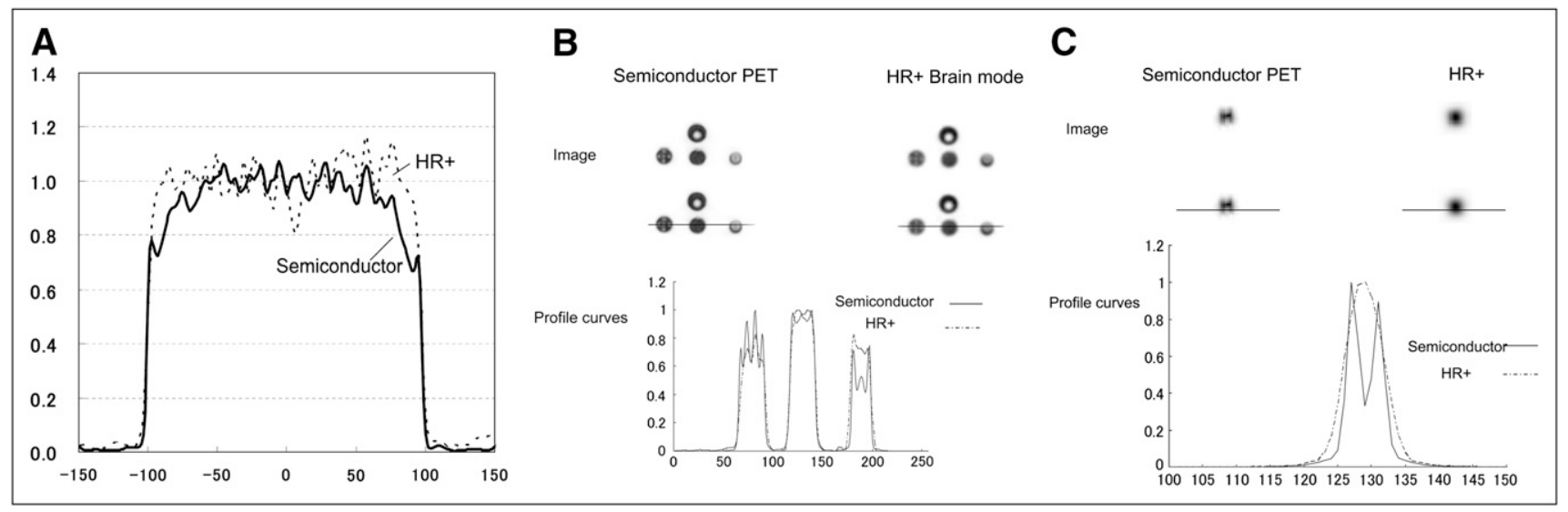

FIGURE 5. Phantom images and profile curves obtained by semiconductor PET and HR+ PET. (A) Pool phantom. (B) Tumor phantom. (C) $\mathrm{H}$-shaped hot phantom. Upper parts of $\mathrm{B}$ and $\mathrm{C}$ show PET images, middle parts show positions of profile curves, and lower parts show profile curves. Profile curves were normalized to maximum counts. 


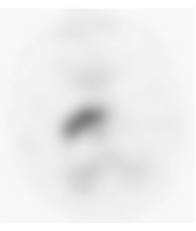

HR+
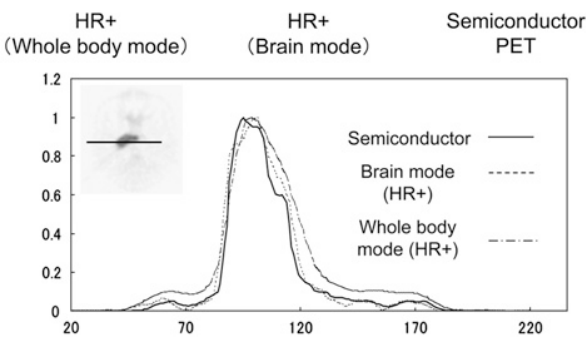

FIGURE 6. Whole-body mode (left) and brain mode (middle) scintillator-based PET images and semiconductor PET image (right) of 61-y-old man with nasopharyngeal squamous cell cancer. Semiconductor PET identified intratumoral inhomogeneous glucose metabolism in more detail than HR+ PET. T2-weighted MRI (T2WI) also revealed inhomogeneous intensity in primary lesion (far right). Diagrams show profile curves. Profile curves were normalized to maximum counts. Profile curve for tumor obtained by semiconductor PET was sharpest among 3 images.

\section{DISCUSSION}

This is the first clinical report of the application of semiconductor PET. Because this PET scanner provides high spatial resolution and low scatter noise, it has the potential for better delineation of intratumoral inhomogeneity in studies of nasopharyngeal cancer. The phantom study confirmed

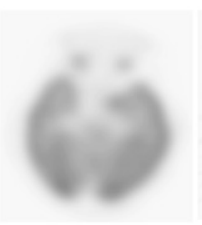

Scintillator PET (Whole body mode)

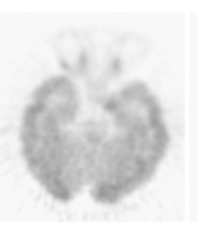

Scintillator PET (Brain mode)

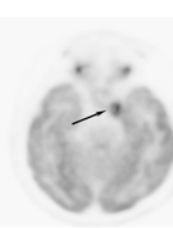

Semiconductor PET

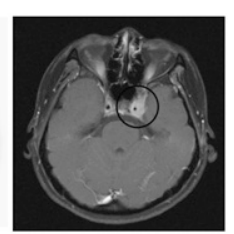

Gd enhanced MRI

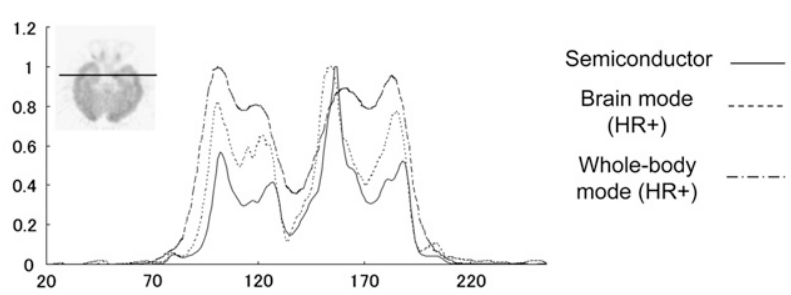

FIGURE 7. Whole-body mode (left) and brain mode (middle) scintillator-based PET images and semiconductor PET image (right) of 35-y-old woman with nasopharyngeal squamous cell cancer. Semiconductor PET revealed low uptake in tumor, which corresponded to internal carotid artery (arrow). Gadolinium-enhanced MRI showed that left internal carotid artery penetrated tumor (far right). Diagrams show profile curves. Profile curves were normalized to maximum counts. Profile curve of tumor obtained by semiconductor PET was sharpest among 3 images.
TABLE 3. Intratumoral Inhomogeneity

\begin{tabular}{|c|c|c|c|}
\hline \multirow[b]{2}{*}{ Patient } & \multicolumn{2}{|c|}{ HR + PET grade } & \multirow[b]{2}{*}{$\begin{array}{l}\text { Semiconductor } \\
\text { PET grade }\end{array}$} \\
\hline & $\begin{array}{l}\text { Whole-body } \\
\text { mode }\end{array}$ & $\begin{array}{l}\text { Brain } \\
\text { mode }\end{array}$ & \\
\hline 1 & 1 & 2 & 3 \\
\hline 2 & 1 & 2 & 3 \\
\hline 3 & 1 & 2 & 3 \\
\hline 4 & 1 & 2 & 3 \\
\hline 5 & 1 & 2 & 3 \\
\hline 6 & 1 & 2 & 3 \\
\hline 7 & 1 & 2 & 3 \\
\hline 8 & 1 & 2 & 3 \\
\hline 9 & 0 & 0 & 0 \\
\hline 10 & 0 & 0 & 0 \\
\hline Mean $\pm S D$ & $0.8 \pm 0.4$ & $1.6 \pm 0.8^{*}$ & $2.4 \pm 1.2^{*}$ \\
\hline $\begin{array}{c}{ }^{*} P<0.01 \\
\text { Grading: } 0 \\
\text { intermediate; } 3\end{array}$ & $\begin{array}{l}\text { no uptake; } \\
\text { nost inhomo }\end{array}$ & $\begin{array}{l}\text { = most } \\
\text { eneous. }\end{array}$ & mogeneous; $2=$ \\
\hline
\end{tabular}

higher contrast obtained for small cold and hot spots with semiconductor PET, with uniformity similar to that obtained with conventional $(\mathrm{HR}+)$ PET. In the study of our specially designed $\mathrm{H}$-shaped hot-rod phantom, a 2-mm defect between 1-mm hot rods was identified by semiconductor PET. In a clinical setting, intratumoral inhomogeneity and edge sharpness were better delineated by semiconductor PET than by HR + PET. This better delineation of intratumoral inhomogeneity is considered to be attributable to the higher spatial resolution and lower scatter noise of our semiconductor PET scanner and not to higher statistical noise.

A semiconductor detector should have 2 significant advantages when it is used as a PET detector. The first advantage is energy resolution. The photopeak of a semiconductor detector is generally sharper than that of a scintillation detector (4). Our new semiconductor PET detectors had an energy resolution of only $4.1 \%$ (FWHM), and our CdTe detectors had almost the same timing resolution $(6.8 \mathrm{~ns}$ [FWHM]) as scintillator-based PET detectors $(15,17)$. The limited energy window set permits the collection of true signal counts with lower noise counts. Therefore, our PET scanner with semiconductor detectors provides high contrast with less scatter noise. As shown in Table 1, the scatter fraction was $23 \%$, which was lower than those of other, scintillation-based PET scanners (14).

The second advantage is superior individual readout. With a semiconductor detector, it is easy to fabricate densely packed detectors that are composed of a large number of small crystal arrays and their amplifiers. Thus, one detector is connected to one amplifier individually. The semiconductor provides a digital signal for each position (18). That is, a separate detector corresponds to each line of response at each position. Consequently, the intrinsic spatial resolution of a semiconductor detector unit is higher than that of a traditional PET detector unit with a positional computer (19). A DOI detection system, which has been used in some scintillator- 
based PET scanners, may reduce parallax errors at the periphery of the FOV in PET scanners dedicated to human brain imaging $(4,5)$. A DOI system and our small detectors were applied to our PET scanner to obtain a higher spatial resolution. In fact, the intrinsic spatial resolution of our PET scanner was $2.3 \mathrm{~mm}$ at $1 \mathrm{~cm}$ or $4.8 \mathrm{~mm}$ at $10 \mathrm{~cm}$. This spatial resolution was higher than that of a conventional PET camera and almost same as that of EXACT HRRT (Siemens Medical Solutions) in a transaxial slice $(14,20)$. Our PET scanner with semiconductor detectors and the DOI system has a high spatial resolution and low scatter noise.

Various components, including nonmalignant components, are involved in most solid tumors (21-28). Various properties of tumor tissues also show intratumoral heterogeneity, which may originate from the diverse phenotypic properties of tumor cells or may be induced by their metabolic microenvironment. In this regard, intratumoral heterogeneity assessed by ${ }^{18}$ F-FDG distribution has been well demonstrated by autoradiography (23-28). Intratumoral ${ }^{18}$ F-FDG distribution has been described at the cellular level by several investigators. Using a malignant tumor mouse model, Kubota et al. showed that ${ }^{18} \mathrm{~F}-\mathrm{FDG}$ preferentially accumulates in macrophages and young granulation tissues surrounding necrotic foci rather than in tumor cells (25). In contrast, Brown et al. observed relatively less ${ }^{3} \mathrm{H}-\mathrm{FDG}$ accumulation in necrotic or inflammatory infiltrates than in tumor cells (26). Semiconductor PET with the DOI system may reveal intratumoral heterogeneity even in a clinical setting.

One of the major limitations of semiconductor PET is the relatively low sensitivity of the semiconductor detector. Low sensitivity is mainly attributable to a narrow energy window setting (490-530 keV) and a low interaction of $511-\mathrm{keV}$ $\gamma$-rays and CdTe materials. To increase the system sensitivity, we used 6-layer detectors in a 3-layer DOI system. In addition, we introduced a new reconstruction algorithm based on MRP and the MAP iterative process. The proposed reconstruction method improved image quality with a high spatial resolution and low statistical noise (16). One may argue that the results for inhomogeneity were attributable to high statistical noise. The uniformity phantom study confirmed that uniformity was well maintained and that uniformity values were similar between semiconductor PET and conventional PET.

It is rather difficult to assess intratumoral inhomogeneity. We applied a 4-point scoring system for objective assessment. Our scoring system showed good interobserver agreement. In addition, our scoring system indicated better identification of intratumoral inhomogeneity by semiconductor PET than by conventional PET. Furthermore, the results for 2 representative patients confirmed the better delineation of intratumoral inhomogeneity corresponding to MRI findings.

Another limitation is that we did not compare our new PET system with a state-of-the-art PET system but rather compared it with a relatively old camera, the HR + system, with standard ordered-subset expectation maximization and directinversion Fourier transformation reconstruction. However, the $\mathrm{HR}+$ system provides relatively high-resolution PET images with the current reconstruction algorithm. We consider it necessary to compare a state-of-the-art lutetium oxyorthosilicate PET scanner with our new PET scanner in the future.

Furthermore, the current brain-dedicated PET camera is a relatively small-aperture instrument. We performed tumor imaging in patients with nasopharyngeal cancer. However, a whole-body scanner is required for most oncologic studies, particularly for assessment of tumor inhomogeneity. We are now planning to develop the next prototype PET camera, with a wide aperture and high sensitivity.

If a whole-body scanner with semiconductor detectors is developed, then application to radiation therapy is expected. Several studies have shown the impact of ${ }^{18}$ F-FDG PET on radiation treatment volumes $(8-11)$. Using a PET scanner with semiconductor detectors and the DOI system, we may be able to perform precise radiation planning to accurately deliver high-dose radiation to lesions with high tumor cell activity.

\section{CONCLUSION}

The phantom and clinical studies suggested that the semiconductor PET scanner has the potential for better identification of intratumoral inhomogeneity because of its higher spatial resolution and lower scatter noise.

\section{REFERENCES}

1. Kubo N, Zhao S, Fujiki Y, et al. Evaluating performance of a pixel array semiconductor SPECT system for small animal imaging. Ann Nucl Med. 2005;19:633-639.

2. Kiyono Y, Kuge Y, Katada Y, Kawashima H, Magata Y, Saji H. Applicability of a high-resolution small semiconductor gamma camera to small animal imaging. Nucl Med Commun. 2007;28:736-741.

3. Scheiber C. CdTe and CdZnTe detectors in nuclear medicine. Nucl Instr Meth A. 2000;448:513-524.

4. Schmand M, Eriksson L, Casey ME, et al. Performance results of a new DOI detector block for a highresolution PET-LSO research tomograph HRRT. IEEE Trans Nucl Sci. 1998;45:3000-3006.

5. Yamaya T, Hagiwara N, Obi T, et al. Preliminary resolution performance of the prototype system for a 4-layer DOI-PET scanner: jPET-D4. IEEE Trans Nucl Sci. 2006;53:11123-11128.

6. Shirato H, Oita M, Fujita K, Watanabe Y, Miyasaka K. Feasibility of synchronization of real-time tumor-tracking radiotherapy and intensity-modulated radiotherapy from viewpoint of excessive dose from fluoroscopy. Int $J$ Radiat Oncol Biol Phys. 2004;60:335-341.

7. Lee N, Puri DR, Blanco AI, Chao KS. Intensity-modulated radiation therapy in head and neck cancers: an update. Head Neck. 2007;29:387-400.

8. Schwartz DL, Ford EC, Rajendran J, et al. FDG-PET/CT-guided intensity modulated head and neck radiotherapy: a pilot investigation. Head Neck. 2005;27:478-487.

9. Wang D, Schultz CJ, Jursinic PA, et al. Initial experience of FDG-PET/CT guided IMRT of head-and-neck carcinoma. Int J Radiat Oncol Biol Phys. 2006;65: $143-151$.

10. Vanderstraeten B, Duthoy W, De Gersem W, De Neve W, Thierens H. $\left[{ }^{18} \mathrm{~F}\right]$ fluoro-deoxy-glucose positron emission tomography $\left(\left[{ }^{18} \mathrm{~F}\right] \mathrm{FDG}-\mathrm{PET}\right)$ voxel intensity-based intensity-modulated radiation therapy (IMRT) for head and neck cancer. Radiother Oncol. 2006;79:249-258.

11. Nishioka T, Shiga T, Shirato H, et al. Image fusion between ${ }^{18}$ FDG-PET and MRI/CT for radiotherapy planning of oropharyngeal and nasopharyngeal carcinomas. Int J Radiat Oncol Biol Phys. 2002;53:1051-1057. 
12. Seino T, Takahashi I. CdTe detector characteristics at $30 \mathrm{C}$ and $35 \mathrm{C}$ when using the periodic bias reset technique. IEEE Trans Nucl Sci. 2007;54:777-781.

13. Herzog H, Tellmann L, Hocke C, Pietrzyk U, Casey M, Kuwert T. NEMA NU22001 guided performance evaluation of four Siemens ECAT PET scanners. IEEE Trans Nucl Sci. 2004;51:2662-2669.

14. Daube-Witherspoon ME, Karp JS, Casey ME, et al. PET performance measurements using the NEMA NU 2-2001 standard. J Nucl Med. 2002;43: 1398-1409.

15. Yanagishita N, Morimoto Y, Ishitsu T, et al. Physical performance of a prototype 3D PET scanner using CdTe detectors. Nuclear Science Symposium Conference Record, 2007. Vol 4. Piscataway, NJ: IEEE; 2007:2665-2668.

16. Takeuchi W, Morimoto Y, Suzuki A, et al. Iterative reconstruction method using prior information and point spread function for high-resolution CdTe PET scanner [abstract]. J Nucl Med. 2008;49(suppl 1):388P.

17. Morimoto $\mathrm{Y}$, Ueno $\mathrm{Y}$, Kobashi K, et al. Performance of a prototype brain PET scanner based on semiconductor detectors [abstract]. J Nucl Med. 2008; 49(suppl 1):122P

18. Ketchum LE. New equipment in nuclear medicine, part 1: solid-state detectors. J Nucl Med. 1998;39(11):15N.

19. Kubo N, Mabuchi M, Katoh C, et al. Validation of left ventricular function from gated single photon computed emission tomography by using a scintillatorphotodiode camera: a dynamic myocardial phantom study. Nucl Med Commun. 2002;23:639-643.

20. Sossi V, de Jong HWAM, Barker WC, et al. The second generation HRRT: a multi-centre scanner performance investigation. Nuclear Science Symposium Conference Record, 2005. Vol 4. Piscataway, NJ: IEEE; 2005:2195-2199.
21. Tovi M, Hartman M, Lilja A, Ericsson A. MR imaging in cerebral gliomas: tissue component analysis in correlation with histopathology of whole-brain specimens. Acta Radiol. 1994;35:495-505.

22. Dachman AH, Pakter RL, Ros PR, Fishman EK, Goodman ZD, Lichtenstein JE. Hepatoblastoma: radiologic-pathologic correlation in 50 cases. Radiology. 1987; 164:15-19.

23. Zhao S, Kuge Y, Mochizuki T, et al. Biologic correlates of intratumoral heterogeneity in ${ }^{18} \mathrm{~F}-\mathrm{FDG}$ distribution with regional expression of glucose transporters and hexokinase-II in experimental tumor. $J$ Nucl Med. 2005;46:675-682.

24. Kubota R, Yamada S, Kubota K, Ishiwata K, Tamahashi N, Ido T. Intratumoral distribution of fluorine-18-fluorodeoxyglucose in vivo: high accumulation in macrophages and granulation tissues studied by microautoradiography. $\mathrm{J} \mathrm{Nucl}$ Med. 1992;33:1972-1980.

25. Kubota R, Kubota K, Yamada S, Tada M, Ido T, Tamahashi N. Microautoradiographic study for the differentiation of intratumoral macrophages, granulation tissues, and cancer cells by the dynamics of fluorine-18-fluorodeoxyglucose uptake. J Nucl Med. 1994;35:104-112.

26. Brown RS, Leung JY, Fisher SJ, Frey KA, Ethier SP, Wahl RL. Intratumoral distribution of tritiated fluorodeoxyglucose in breast carcinoma. I. Are inflammatory cells important? J Nucl Med. 1995;36:1854-1861.

27. Kubota R, Kubota K, Yamada S, Tada M, Ido T, Tamahashi N. Active and passive mechanisms of [fluorine-18]fluorodeoxyglucose uptake by proliferating and prenecrotic cancer cells in vivo: a microautoradiographic study. $J$ Nucl Med. 1994;35:1067-1075.

28. Kubota R, Kubota K, Yamada S, et al. Methionine uptake by tumor tissue: a microautoradiographic comparison with FDG. J Nucl Med. 1995;36:484-492. 